\title{
Synchrotron Radiation Techniques in Catalytic Science
}

\author{
C.Richard A Catlow ${ }^{\mathrm{a}, \mathrm{b}, \mathrm{c}}$, Peter Wells ${ }^{\mathrm{c}, \mathrm{d}, \mathrm{e}}$, Diego Gianolio \\ a Department of Chemistry, University College London, 20 Gordon St., London WC1 HOAJ \\ b School of Chemistry, Cardiff University, Park Place, Cardiff CF10 3AT \\ c UK Catalysis Hub, Research Complex at Harwell, Harwell, Didcot, Oxfordshire, OX11 OFA \\ d School of Chemistry, University of Southampton, University Road, Southampton, SO17 1BJ \\ e Diamond Light Source, Harwell Science and Innovation Campus, Didcot, Oxfordshire, OX11 ODE
}

Catalysis is a core area of current chemical and engineering sciences, which while posing fundamental scientific challenges is also a corner stone of the chemicals and pharmaceuticals industries underpinning a vast range of products and processes worldwide. Developments in catalytic technology including the design and optimisation of catalysts and catalytic processes rely increasingly on the development of an understanding of catalysis at the molecular level. In developing molecular level models of catalytic processes, techniques employing Synchrotron Radiation (SR) have had a transformative effect on catalytic science. The unique properties of SR have led to entirely new opportunities in diffraction, spectroscopy, small angle scattering and tomographical studies of catalytic materials. Moreover, SR has been crucial in enabling the growth of in situ experimental studies of catalytic processes under realistic operating conditions. The field impacts on all areas of catalytic science, including heterogeneous, homogeneous, bio-catalysis and chemical engineering aspects.

Catalytic science has exploited SR since the early days of the field in the 1970 s and ' 80 s. Most early work employed X-Ray spectroscopy, in particular the ability of X-Ray Absorption Fine Structure (XAFS) to provide element specific local structural information, which as such is able to provide unique information on active site structures. Over the last decade, the use of XAFS within the field has moved beyond the preserve of specialist groups to become an essential tool in characterising advanced functional materials. The wealth of XAFS based studies within this special issue provides demonstrable evidence of this burgeoning user community. This leads us to two important questions: i) what next for XAFS studies within catalytic science, and ii) how can other SR methods use this experience to develop further?

Regarding XAFS, there are many emerging technological developments. The advances in synchrotron lattice, optics and detectors over recent years have led to improved beam brilliance, spatial and temporal resolution. These improvements have resulted in a higher selectivity and sensitivity of the technique. Quick EXAFS scanning has been particularly beneficial for the study of reactions such as photocatalysis and electrochemistry. The smaller beam spot has opened the possibility of sampling heterogeneous materials in different points, for example highlighting differences in reaction under study in different points of the catalytic bed. The higher flux provided by the new $4^{\text {th }}$ generation synchrotrons will also open new possibilities for a more extensive use of photon-hungry techniques such as X-ray emission spectroscopies, reducing the measurement times and therefore allowing in situ experiments that are still challenging. X-ray Raman is another rapidly advancing method that will also benefit from the higher brilliance and will allow the use of hard X-rays to measure soft edges overcoming the problems of strong absorption from sample environment, windows and liquid media, offering extended capabilities for in situ characterization of homogeneous catalysts and the study of electrochemistry/batteries. Elsewhere, as demand for conventional and advanced XAFS spectroscopy increases further, there will be changes in the way 
measurements are performed. There have been significant advances in sample automation and lab-based instruments, which will be imperative for the future as demand continues to increase.

SR methods are not limited to XAFS, a good example being High Resolution $X$-Ray Powder Diffraction (XRPD), which when coupled with Rietveld refinement techniques enabled the determination of high quality crystal structures for complex catalytic systems, especially microporous materials. The current issue again provides good illustrations of the role of XRPD in the field. The recent rise of the X-ray pair distribution function (XPDF) method has provided a missing link between XRD and XAS and helped the combined refinement of datasets, therefore building a more thorough knowledge of the materials investigated. The development of microcrystalline, single crystal diffraction techniques has also played a significant role in the structural chemistry of microporous catalysts.

Other more recent developments include the growth of tomographic imaging techniques and the use of SR based infra-red spectroscopy, and the possibility of running ambient pressure XPS experiments that allow surface sensitive experiments on catalysts under in situ conditions at soft edges. It is clear that these methods can benefit from the experience of other techniques; in particular, the use of shared access methods has revolutionised the uptake of new users - and different chemistry - as well as providing a focal point on which to nurture the community. Moreover, the technical developments in synchrotron machines and techniques have been enhanced by the parallel growth of theory and data analysis methods such as wavelet transform, neural networks and machine learning applied to SR data. These upgrades provide better tools for the interpretation of the data collected and help the user community to gain a deeper understanding of the systems studied.

The field is developing rapidly and even greater impact of synchrotron based techniques on catalytic science can be expected in the future. We hope that this issue gives a good illustration both of the current range of catalytic science enabled by these exceptional radiation sources, while pointing to the future possibilities 